\title{
Magnesium intervention and blood pressure-A study on risk groups
}

\author{
Ragnar Rylander ${ }^{1}$, Tommi Tallheden ${ }^{2}$, Jürgen Vormann ${ }^{3}$ \\ ${ }^{1}$ BioFact Environmental Health Research Center, Lerum, Sweden; envhealth@biofact.se \\ ${ }^{2}$ Department of Clinical Chemistry, Skene Hospital, Skene, Sweden; tommi.tallheden@vgregion.se \\ ${ }^{3}$ Institute for Prevention and Nutrition, Ismaning, Germany; vormann@ipev.de
}

Received 13 October 2011; revised 23 November 2011; accepted 24 December 2011

\begin{abstract}
Hypothesis: Although mineral deficiency in particular magnesium is a known risk factor for cardiovascular disease intervention experiments with magnesium show only modest results. Previously published data suggest that there may be particular risk groups in the population. The study was undertaken to investigate if such risk groups could be identified. Experimental design: Subjects $(n=$ 31) were 50 - 79 years old with no disease. The 24 hour urinary excretion of urea (proxy for acidity), magnesium, calcium, and potassium was measured at two week intervals, with and without intervention with mineral water. Intervention with mineral water comprised $75 \mathrm{~mL}$ daily, supplying 3.1 $\mathrm{mmol} \mathrm{Mg}, 2.3 \mathrm{Ca}$, and $0.02 \mathrm{~K}$. Major results: There was a significant relation between acidity and excretion of magnesium, calcium, and potassium, both at one occasion and regarding changes over time. Among subjects with a high secretion of urea there was an inverse relation between the excretion of magnesium and systolic blood pressure. After intervention with mineral water, there was a strong tendency to a decrease in the systolic blood pressure among those with an initial high excretion of urea and a low excretion of magnesium. Conclusions: The results demonstrate the importance of acid-base conditions for mineral homeostasis and suggest the presence of risk groups in the population, related either to a low intake or to a disorder in the reabsorbtion mechanisms. Mineral water decreased the systolic blood pressure in that particular risk group.
\end{abstract}

Keywords: Acid-Base; Magnesium; Calcium; Mineral Water

\section{INTRODUCTION}

Cardiovascular diseases are a large public health problem particularly in Western countries and preventive actions have a high priority from a public health point of view. There is considerable evidence that various minerals, particularly magnesium play an important role in the pathogenesis of these diseases through an influence on myocardial function [1-3]. Low serum levels of magnesium have been related to an increased risk of sudden death [4]. Several studies have shown a relation between drinking water magnesium and risk of cardiovascular disease, particularly death in heart infarction [5,6]. The relevant literature was subject to a meta-analysis which concluded "the evidence from this review supports an association between cardiovascular mortality and magnesium levels in water" [7].

Several studies have been performed where the influence on blood pressure of an oral administration of magnesium was studied [review in 8]. Although some studies report a beneficial effect, it is relatively small and not consistent. In a previous study a significant decrease in blood pressure was found after intervention with mineral water but only among subjects with an initial low urinary excretion of magnesium [9]. This suggests the presence of a risk group in the population with regard to magnesium homeostasis and blood pressure increase. This risk group concept is supported by a study in the Netherlands, where a relation was found between stroke mortality and the magnesium content in drinking water but only among males with a dietary inadequate intake of magnesium [10]. Further support comes from a study with magnesium intervention for blood pressure [11]. A study on British adults found that the normal dietary sources in that population were inadequate for magnesium, suggesting that there could be particular risk groups in the population related to dietary habits, akin to the findings in the Netherlands study [10]. The outcome of studies on the effect of magnesium on cardiovascular disease could thus depend upon the presence and the size of risk groups in the studied population.

The magnesium homeostasis in the body is, apart from intake through food and water, regulated by acid-base conditions. A study on a population sample demonstrated a close association between the net acid excretion (NAE) 
and the amounts of magnesium and calcium in the urine [13]. These data suggest that mineral deficiency may develop, not only because of an insufficient intake, but also because of acid conditions in the body, which prevent reabsorption of minerals in the kidneys. The results from a study comparing different age groups suggest that the elderly are a risk group for mineral deficiency due to acid conditions in the body [14].

The present study represents an attempt to identify a risk group for magnesium deficiency in relation to acid-base conditions. The aims were to

1) further explore the relation between the acid-base balance and urinary excretion of minerals in a randomly chosen population group;

2) explore the presence of particular groups at risk for high blood pressure;

3) assess the possibility to intervene in these groups with mineral rich water.

\section{METHODS AND MATERIALS}

\subsection{Population Sample}

The population sample studied comprised voluntary subjects aged 50 - 79 years without disease. Exclusion criteria were medication regulating the cardiovascular or kidney function. There were 31 subjects (mean age 62 years, 62\% females, no smokers). The test sessions were two weeks with examinations before and after this period. In the mineral water sessions, 18 subjects participated once and 10 had two sessions, at least 2 months apart.

The study was conducted according to the guidelines laid down in the Declaration of Helsinki and all procedures involved were approved by the Ethical Committee of Gothenburg, Sweden (Dnr 600-06). Verbal information consent was obtained from all subjects, witnessed, and formally recorded.

\subsection{Sampling}

Measurements were made of the 24 h urinary excretion of urea (as proxy for acid-base balance) [15] and of magnesium, calcium, and potassium. The subjects collected urine samples in their homes and the analyses were performed at an accredited hospital laboratory (Borås, Sweden) using standard techniques.

Systolic and diastolic blood pressures were taken by the same person throughout the study, using an automatic recording devise (Boso, Bosch + Sohn, Jungigen, Germany) in the subject's home with the subjects seated after 5 minutes resting. Two readings were taken and the mean of those was recorded. The measurements were taken at baseline and after 14 days, with and without intervention with mineral water.

\subsection{Intervention}

The mineral water intervention comprised five doses, daily evenly spread over the day and with no specific relation to mealtimes. The total daily dose was about 75 $\mathrm{mL}$. The content of the mineral per daily dose in mmol was $3.1 \mathrm{Mg}, 0.65 \mathrm{Ca}$, and $0.02 \mathrm{~K}$.

\subsection{Statistical Evaluation}

The statistical evaluation was made using non-parametric tests in view of the non-normal distribution of the measured data. Differences between groups were evaluated using the Mann-Whitney test. Relationships between parameters were evaluated using Spearman's test. A p-value of 0.05 was considered statistically significant.

\section{RESULTS}

\subsection{Baseline Conditions}

The relationships between the baseline acidity (excretion of urea) and different minerals was evaluated in 65 observations-17 from sessions with no mineral water intervention and 38 as initial values from sessions with intervention.

The relations between the urinary excretion of urea and all minerals were significant (calcium $\mathrm{p}=0.015$, magnesium 0.020 and potassium $<0.001$ ). There were no relationships between the urea excretion and systolic or diastolic blood pressure.

The relation between urea and potassium is illustrated in Figure 1.

A further analysis was made of the subjects with a urea excretion in excess of the 2nd quartile (393 mmol/24h). In this group there was an inverse relation between the excretion of magnesium and systolic blood pressure (corr coeff-0.469, $\mathrm{p}<0.016)$. There were no relations for

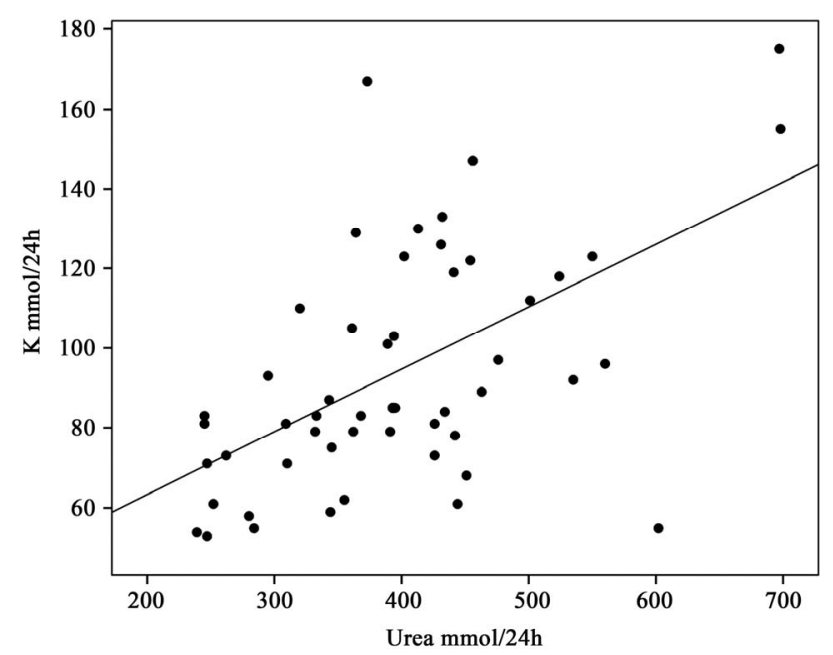

Figure 1. Relation between urinary excretion of urea and potassium. 
calcium or potassium. The relationship is illustrated in $\mathbf{F i -}$ gure 2.

A lower excretion of magnesium was related to a higher blood pressure. There was no relationship for the group with a low excretion of urea ( $<2$ nd quartile).

\subsection{Changes over Time without Intervention}

The relationships between the changes in excretion of urea and different minerals between two sampling occasions in sessions without mineral water intervention were significant for urea and calcium and potassium $(p=0.001$ for both) and close to significance for magnesium ( $\mathrm{p}=$ 0.070). There were no relationships between the changes in urinary secretion and the changes in systolic or diastolic blood pressure.

\subsection{Intervention with Mineral Water-Minerals}

Table 1 reports the mineral excretion before and after intervention with mineral water.

In the group receiving mineral water there was a small and almost significant increase in the secretion of magnesium and calcium ( $\mathrm{p}=0.051$ and 0.075 respectively, Wilcoxon signed rank test). The increase in calcium in the control group was not significant $(\mathrm{p}=0.107)$.

\subsection{Intervention with Mineral Water-Blood Pressure}

In the whole material there were no differences in systolic or diastolic blood pressure after intervention with mineral water (data not shown). Among subjects with a high urea excretion and an excretion of magnesium less than $2.7 \mathrm{mmol} / 24 \mathrm{~h}$ (3rd quartile, see Figure 2) receiving mineral water $(n=5)$, four subjects decreased their diastolic

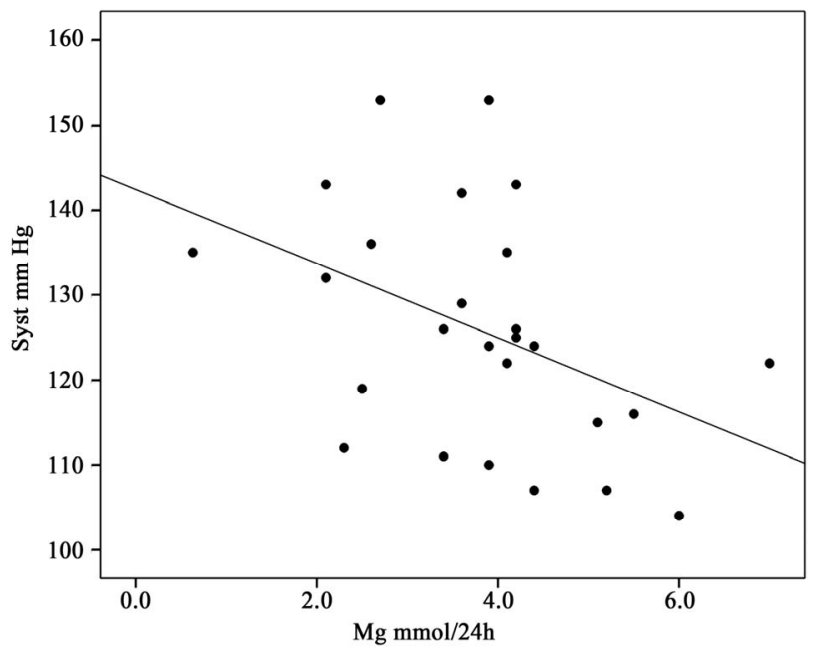

Figure 2. Relation between urinary excretion of magnesium and systolic blood pressure among subjects with a urea excretion >393 mmol (2nd quartile).
Table 1. Mineral excretion (mmol) before and after intervention with mineral water. Mean and standard error of the mean.

\begin{tabular}{|c|c|c|c|}
\hline \multicolumn{2}{|c|}{ Intervention none } & \multicolumn{2}{|c|}{ Water } \\
\hline & $\mathrm{n}$ & 17 & 38 \\
\hline \multirow{2}{*}{ Magnesium } & Before & $3.5(0.2)$ & $3.5(0.2)$ \\
\hline & After & $3.6(0.3)$ & $3.9(0.2)$ \\
\hline \multirow{2}{*}{ Calcium } & Before & $3.1(0.4)$ & $3.3(0.3)$ \\
\hline & After & $3.7(0.5)$ & $3.7(0.5)$ \\
\hline \multirow{2}{*}{ Potassium } & Before & 89 (6.1) & $97(5.0)$ \\
\hline & After & $86(5.7)$ & $93(4.0)$ \\
\hline
\end{tabular}

blood pressure with the average decrease in the group from 78.0 to 73.8 .

\section{COMMENTS}

The results confirm observations from previous studies that acid-base conditions in the body are related to the mineral homeostasis. Also changes in the acid-base balance over a short time period, reflecting variations in the diet, influenced the secretion of magnesium and calcium. There was an inverse relation between magnesium excretion and systolic blood pressure among those with high acidity and indications of an effect of mineral water on the blood pressure in this group.

There are some methodological drawbacks in the study. Acid-base conditions were estimated using the excretion of urea. This excretion, although significantly related to the acid-base balance [15] is not a determinant there off. Changes in the acid-base balance induced by an intervention would not alter the excretion of urea which is determined by metabolism of nutrients, particularly proteins [16]. None of the subjects had kidney disease and the acid conditions, illustrated by the urea secretion, would thus be determined by dietary factors, principally the consumption of proteins.

Another drawback is the population sample. In studies of this kind with voluntary participation, there is a bias in selection as the participants are often health conscious and have a balanced diet. This is reflected by the absence of smokers in the material and the low number of persons with a high urea excretion (Figure 1). No data on subjects' dietary habits etc. were collected as the aim was to investigate a population sample comprising individuals with normal variations in the daily diet. That changes did occur was demonstrated in the changes in excreted urinary minerals on two occasions two weeks apart.

The result that a higher excretion of minerals is present at higher levels of acidity is in agreement with previous studies $[13,15]$. The present study also demonstrated that changes in the system appear within weeks.

There was no general decrease in the excretion of calcium and magnesium after the mineral water intervention. This implies that the effect on the acid-base balance in- 
duced by the mineral water was less than the influence of dietary factors.

Although the number of subjects was low, the data suggest that intervention with mineral water decreased the diastolic blood pressures in the group with a high secretion of urea and a low secretion of magnesium. This is consistent with the presence of a risk group and confirms the findings from a previous study where an effect on blood pressure was seen after mineral water intervention but only in a group with an initial low urinary excretion of magnesium and calcium [9]. This existence of risk groups could also explain the inconsistent outcome of previous intervention studies with magnesium where the presence of such individuals was not controlled for [8].

From a public health point of view, the results suggest that an adjustment of dietary components that decrease the acid load is beneficial for mineral homeostasis and that intervention with an alkaline and magnesium rich mineral water could decrease a slightly elevated blood pressure among subjects with a deficiency in magnesium or with an inadequate magnesium supply.

In conclusion, the study demonstrated a relation between the acid-base balance and the excretion of magnesium, calcium, and potassium and an inverse relation between urinary excretion of magnesium and systolic blood pressure among subjects with a high acid load. The results indicate that intervention with mineral water could decrease the blood pressure in a specific risk group.

\section{ACKNOWLEDGEMENTS}

The study received financial support from Droga Kolinska, Živilska industrija, d.d., Ljubljana, Slovenia.

None of the authors has a conflict of interest.

\section{REFERENCES}

[1] Mathers, T.W. and Beckstrand, R.L. (2009) Oral magnesium supplementation in adults with coronary heart disease or coronary heart disease risk. Journal of the American Academy of Nurse Practitioners, 21, 651-657. doi:10.1111/j.1745-7599.2009.00460.x

[2] Hashimoto, T., Hara, A., Ohkubo, T., Kikuya, M., Shintani, Y., Metoki, H., Inoue, R., Asayama, K., Kanno, A., Nakashita, M., Terata, S., Obara, T., Hirose, T., Hoshi, H., Totsune, K., Satoh, H. and Imai, Y. (2010) Serum magnesium, ambulatory blood pressure, and carotid artery alterations: The Ohasama study. American Journal of Hypertension, 23, 1292-1298. doi:10.1038/ajh.2010.168

[3] Yamon, Y. and Mizushima, S. (2000) A review of the link between dietary magnesium and cardiovascular risk. Journal of Cardiovascular Risk, 7, 31-35.

[4] Peacock, J.M., Ohira, T., Post, W., Sotoodehnia, N., Rosamond, W. and Folsom, A.R. (2010) Serum magnesium and risk of sudden cardiac death in the atherosclerosis risk in communities (ARIC) study. American Heart Journal, 160, 464-470. doi:10.1016/j.ahj.2010.06.012

[5] Rylander, R. (1996) Environmental magnesium deficiency as a cardiovascular risk factor. Journal of Cardiovascular Risk, 3, 4-10. doi:10.1097/00043798-199602000-00002

[6] Rubenowitz, E., Molin, I., Axelsson, G. and Rylander, R. (2000) Magnesium in drinking water in relation to morbidity and mortality from acute myocardial infarction. Epidemiology, 11, 416-421. doi:10.1097/00001648-200007000-00009

[7] Catling, L.A., Abubakar, I., Lake, I.R., Swift, L. and Hunter, P.R. (2008) A systematic review of analytical observational studies investigating the association between crdiovascular disease and drinking water hardness. Journal of Water and Health, 4, 433-342. doi:10.2166/wh.2008.054

[8] Jee, S.H., Miller, E.R., Guallar, E., Singh, V.K., Appel, L.J. and Klag, M.J. (2002) The effect of magnesium supplementation on blood pressure: A meta-analysis of randomized clinical trials. American Journal of Hypertension, 15, 691-696. doi:10.1016/S0895-7061(02)02964-3

[9] Rylander, R. and Arnaud, M.J. (2004) Mineral water intake reduces blood pressure among subjects with low urinary magnesium and calcium levels. BMC Public Health, 4, 56-65. doi:10.1186/1471-2458-4-56

[10] Leurs, L.L., Schouten, L.J., Mons, M.N., Goldbohm, R.A. and van den Brandt, P.A. (2009) Relationship between tap water hardness, magnesium, and calcium concentration and mortality due to ischemic heart disease or stroke in the Netherlands. Environmental Health Perspectives, 118, 414-420. doi:10.1289/ehp.0900782

[11] Lind, L., Lithell, T. and Ljunghall, S. (1992) Blood pressure response during long-term treatment with magnesium is dependent on magnesium status. American Journal of Hypertension, 4, 674-679.

[12] Rouston, D., Hoare, J. and Gregory, J. (2004) The national diet and nutrition survey: Adults aged 19 - 64. The Stationery Office, London.

[13] Rylander, R., Remer, T., Berkemeyer, S. and Vormann, J. (2006) Relationship of magnesium and acid-base balance in an elderly population. European Journal of Nutrition, 36, 2374-2377.

[14] Remer, T., Berkemeyer, S., Rylander, R. and Vormann, J. (2007) Muscularity and adiposity in addition to net acid excretion as predictors of 24-h urinary $\mathrm{pH}$ in young adults and elderly. European Journal of Clinical Nutrition, 61, 605-609.

[15] Rylander, R., Tallheden, T. and Vormann, J. (2009) Acidbase conditions regulate calcium and magnesium homeostasis. Magnesium Research, 22, 1-4.

[16] Remer, T. (2001) Influence of nutrition on acid-base balance-metabolic aspects. European Journal of Nutrition, 40, 214-220. doi:10.1007/s394-001-8348-1 